\title{
OPEN Superhydrophobic functionalized cellulosic paper by copper hydroxide nanorods for oils purification
}

\author{
Ahmed S. Belal ${ }^{1}$, Jehan El Nady ${ }^{2}$, Azza Shokry ${ }^{1}$, Shaker Ebrahim ${ }^{1}$, Moataz Soliman ${ }^{1}$ \& \\ Marwa Khali ${ }^{3 凶}$
}

Oily water contamination has been sighted as one of the most global environmental pollution. Herein, copper hydroxide nanorods layer was constructed onto cellulosic filter paper surface cured with polydopamine, Ag nanoparticles, and Cu NPs through immersion method. This work has been aimed to produce a superhydrophobic and superoleophilic cellulosic filter paper. The structure, crystalline, and morphological properties of these modified cellulosic filter paper were investigated. Scanning electron microscope images confirmed that the modified surface was rougher compared with the pristine surface. The contact angle measurement confirmed the hydrophobic nature of these modified surfaces with a water contact angle of $169.7^{\circ}$. The absorption capacity was $8.2 \mathrm{~g} / \mathrm{g}$ for diesel oil and the separation efficiency was higher than $99 \%$. It was noted that the flux in the case of low viscosity solvent as $\mathrm{n}$-hexane was $9663.5 \mathrm{Lm}^{-2} \mathrm{~h}^{-1}$, while for the viscous oil as diesel was $1452.7 \mathrm{Lm}^{-2} \mathrm{~h}^{-1}$.

The crude oils extracted from oil wells are not pure but are mixed with various liquids and solid matters such as dust, fine sand, sludge, clay, water with dissolved salts, oxides, hydroxides, sulfides, etc. The separation of these impurities is difficult to perform by just one method and the techniques of separation can be based on mechanical, thermal, chemical, or electrical character. These methods are slow, expensive, and inefficient ${ }^{1-3}$. There are porous materials such as sponges, foams, and textiles used to purify the oils from water. However, these materials are suffered from low capacity and rejection efficiency. Moreover, the recycling or reuse of these materials is difficult and time-consuming ${ }^{4-6}$. Consequently, the development of effective oil/water separation cost-effective, eco-friendly, and recyclable superhydrophobic nanomaterials with high efficiency and flux are in high demand ${ }^{7,8}$.

Superhydrophobic materials have been used for oil/water separation as metal ${ }^{9}$, fabric $^{6,10,11}$ sponge $^{12,13}$, paper $^{14}$, etc. cellulosic filter paper has been received concern due to its porous structure made of microfibers and low cost. However, only a few reports of the superhydrophobic cellulosic filter paper including hydrogel-coated ${ }^{15}$, dip-coating ${ }^{16}$, and PDVB-PDMS decorated ${ }^{17}$ were applied in the emulsion separation. Developing green and low-cost materials by facile preparation approach for efficient separation is instantly required ${ }^{18,19}$.

This work is aimed to functionalize the cellulosic filter paper top surface with $\mathrm{Cu}(\mathrm{OH})_{2}$ nanostructures. The modification on this cellulosic filter paper is carried out by coating $n$-dodecanethiol to reduce the surface energy. In addition, we concern with both oil/water separation technologies based on the filtration and absorption methods. The different parameters such as separation efficiency, flux, and oil absorption capacity of this modified cellulosic paper filter for chloroform, dichloromethane, toluene, $n$-hexane, diesel, sunflower oil, olive oil, waste engine oil, and diesel oil are evaluated and compared.

\section{Materials and methods}

Materials. Cellulosic filter paper composed from high-quality cotton liners, which have been treated to achieve a minimum alpha cellulose content of $98 \%$ was bought from German (Whatman style filter paper is made from cellulose and is used for general filtration) and dopamine hydrochloride was obtained from Sunny

\footnotetext{
${ }^{1}$ Materials Science Department, Institute of Graduate Studies and Research, Alexandria University, P.O. Box 832, Alexandria, Egypt. ${ }^{2}$ Electronic Materials Department, Advanced Technology and New Materials Research Institute, City of Scientific Research and Technological Applications (SRTA-City), New Borg El-Arab City, P.O. Box 21934, Alexandria, Egypt. ${ }^{3}$ Nanotechnology and Composite Materials Department, Institute of New Materials and Advanced Technology, City of Scientific Research and Technological Applications (SRTA-City), New Borg El Arab City, P.O. Box 21934, Alexandria, Egypt. ${ }^{\square}$ email: igsr.marwa.khalil@alexu.edu.eg
} 
Pharmaceutical Company. Ammonium hydroxide (25\%) was obtained from Sigma. Silver nitrate (99.8\%), sodium hydroxide (96\%), and dichloromethane were purchased from Merk. Glucose was received from El Nasr Pharmaceutical Chemical Company. Copper sulfate pentahydrate (99\%) and ethanol were bought from Fisher Scientific. Potassium sodium tartrate (99\%) ammonium persulfate (98\%), chloroform, toluene, and n-hexane were brought out from BDH company. Dodecanethiol (98\%,) was purchased from Across Company. Diesel, sunflower oil, and olive oil were obtained from the local market.

Preparation of copper hydroxide nanorods. Firstly, the cellulosic filter paper was washed with a mixture of deionized water and ethanol and consequently, it was modified by in situ polymerization of dopamine hydrochloride by immersing the cellulosic paper in the prepared $2 \mathrm{mg} / \mathrm{mL}$ dopamine solution with $\mathrm{pH} 8.5$ (adjusted with ammonia) for $24 \mathrm{~h}$ at $60^{\circ} \mathrm{C}$ with continuous stirring. This modified cellulosic paper was washed with deionized water. This modified surface of the cellulosic paper was treated with $0.05 \mathrm{M}$ of $\mathrm{AgNO}_{3}$ and $0.01 \mathrm{M}$ glucose with sustaining $\mathrm{pH}$ to 8.0 for $6 \mathrm{~h}$. Finally, this modified cellulosic paper was washed with deionized water and dried at room temperature.

The $\mathrm{Cu}$ NPs layer onto Ag NPs modified cellulosic paper was dipped in a mixture of $80 \mathrm{~mL}$ Fehling B reagent solution. Moreover, the copper hydroxide layer was produced by immersing the last $\mathrm{Cu} / \mathrm{Ag}$ NPs modified cellulosic paper in $1 \mathrm{M} \mathrm{NaOH}$ and $0.05 \mathrm{M}$ ammonium persulfate for $30 \mathrm{~min}$. The surface was also rinsed and washed with deionized water and dried at ambient temperature for $24 \mathrm{~h}$. In the final step, the modified cellulosic surface was functionalized with an ethanolic solution of $0.01 \mathrm{M} \mathrm{n}$-dodecanethiol for $30 \mathrm{~min}$.

Characterization techniques. The structural formed layers onto the cellulosic filter paper were characterized by Fourier transform infrared (FTIR) spectrophotometer (Spectrum BX 11- Perkin Elmer). The FTIR spectra were measured in the range from 4000 to $400 \mathrm{~cm}^{-1}$. Each layer was grinded with $\mathrm{KBr}$ powder and this powder was pressed to form a translucent pellet.

Copper hydroxide was characterized by Raman spectra which was recorded using a microscope equipped with triple monochromatic combined with a peltier cooled charge couple device detector system (Senterra Bruker). The spectra were acquired in the back-scattering geometry while the $532 \mathrm{~nm}$ line of an Argon laser source was focused on the sample for excitation at a power of $2 \mathrm{~mW}$. The phonon frequencies were obtained by fitting Lorentzian line shapes to the experimental peaks after background subtraction.

The absorption spectrum of the suspension solution of $\mathrm{PD} / \mathrm{Ag} / \mathrm{Cu}$ and $\mathrm{PD} / \mathrm{Cu}$ was recorded with a UV-visible spectrophotometer (Evolution 300, Thermoscientifc, USA). The measurement was performed in quartz cuvettes using $\mathrm{H} 2 \mathrm{O}$ as a solvent. The light source wavelengths were scanned in the range of 200 to $600 \mathrm{~nm}$.

The XRD analysis of the copper hydroxide formed onto the cellulosic filter paper surface was evaluated by Bruker- AXS D8 Discover. The Bragge's angle (20) in the range from 5 to 80 degrees was varied. The X-ray source of $\mathrm{Cu}$ target was used at $30 \mathrm{kV}$ and $30 \mathrm{~mA}$. The morphology of the modified cellulosic filter paper was examined by SEM attached with energy dispersive X-ray (EDX) ("JEOL JSM 6360LA", Japan). The modified cellulosic filter paper layers were sputtered with $\mathrm{Au}$ film. $\mathrm{Cu}(\mathrm{OH})_{2}$ nanostructure was confirmed with high-resolution transmission electron microscope (HRTEM) attached with selected area electron diffraction (SAED) (JEOL, JEM-2100 LaB6).

The hydrophilicity and hydrophobicity of the modified cellulosic filter paper were evaluated by contact angle measurements using Rame-hart, France. The average of the 5 measurements at different positions was recorded.

The performance of modified cellulosic filter paper for oils and organic solvents removal. The absorption process was measured for different viscous oils (diesel engine oil, waste engine oil, olive oil, and sunflower oil) and organic solvents such as n-hexane, dichloromethane, chloroform, and toluene. The absorption capacity ( $\mathrm{Q}$ in $\mathrm{g} / \mathrm{g}$ ) was calculated by the following equation ${ }^{4}$ :

$$
Q=\frac{m_{e}-m_{o}}{m_{o}}
$$

where $\boldsymbol{m}_{\mathrm{o}}$ is the initial weight of the modified cellulosic filter paper and $\boldsymbol{m}_{\mathrm{e}}$ is the weight of the modified cellulosic filter paper after immersing in oils or organic solvents and water.

The modified surface of cellulosic filter paper was used as a filter for oil (organic solvents)/water separation. Oils or organic solvents/water mixtures with the volume ratio of 1:1 (50 mL of dyed oil with blue ink and $50 \mathrm{~mL}$ of dyed water with green ink) was used. This modified cellulosic filter paper was fixed at a separation system and serving as a separation membrane. The reused of the modified cellulosic filter paper was repeated for more than 100 cycles. The efficiency of separation of oils or organic solvents/water mixtures was estimated from the following Eq. ${ }^{20}$ :

$$
\text { Separation Efficiency }(\%)=\frac{\boldsymbol{m}_{1}}{\boldsymbol{m}_{2}} * 100
$$

where $\boldsymbol{m}_{\mathbf{1}}$ and $\boldsymbol{m}_{\mathbf{2}}$ are the weight of the collected oil and the initial weight of oil, respectively. The flux of oils (organic solvents) in $\mathrm{L} \mathrm{m}^{-2} \mathrm{~h}^{-1}(\mathrm{~V})$ was calculated from the volume of the oils or organic solvents from the following equation ${ }^{20}$ :

$$
F l u x=\frac{V}{S t}
$$

where $S$ is the surface area of cellulosic filter paper and $\boldsymbol{t}$ is the separation time. 


\section{Results and discussion}

Structural of the modified surface of cellulosic filter paper. The cellulosic feature is confirmed by FTIR spectra for the pristine and modified cellulosic filter paper as shown in Fig. 1a. The broad absorption band at $3400 \mathrm{~cm}^{-1}$ is matched with $\mathrm{O}-\mathrm{H}$ stretching vibrations arising from hydroxyl and hydrogen-bonded hydroxyl groups ${ }^{21}$. The peak at $1635 \mathrm{~cm}^{-1}$ assigns to $\mathrm{O}-\mathrm{H}$ bending vibration of the adsorbed water 22,23 . The bands of antisymmetrical C-O and C-O-C stretching vibrations of cellulose appear at 1132 and $1025 \mathrm{~cm}^{-1}$, respectively ${ }^{24}$. The peak at $1430 \mathrm{~cm}^{-1}$ is related to $\mathrm{C}-\mathrm{H}$ scissoring motion of cellulose. The absorption bands at $2900 \mathrm{~cm}^{-1}$ and $1340 \mathrm{~cm}^{-1}$ are assigned to $\mathrm{C}-\mathrm{H}$ stretching and bending vibrations, respectively. The polymerization of the polydopamine layer onto cellulosic filter paper surface is confirmed by indole or indoline moieties and hydroxyl groups that appear at $1637 \mathrm{~cm}^{-1}$ and $3300-3550 \mathrm{~cm}^{-1}$, respectively ${ }^{21,25,26}$. The bands of the infrared spectrum of cellulosic filter paper coated with PD/Ag NPs layers are slightly shifted. The formation of Ag NPs layer does not affect the surface chemical structure of polydopamine ${ }^{27}$. The peak at $3550 \mathrm{~cm}^{-1}$ attributed to the stretching vibrations of $-\mathrm{OH}$ and $\mathrm{N}-\mathrm{H}$ groups in the polydopamine is broadened due to the formation of the hydrogen bond between PD and Ag NPs layers. The peaks at $1604 \mathrm{~cm}^{-1}, 1512 \mathrm{~cm}^{-1}$, and $1367 \mathrm{~cm}^{-1}$ are recognized to the stretching vibrations of $-\mathrm{C}=\mathrm{C}-,-\mathrm{C}=\mathrm{N}$ and $\mathrm{C}-\mathrm{N}-\mathrm{C}$ functional groups associated with indoline (or) indole structure present polydopamine ${ }^{28}$. The good stability of the hierarchical structure of the celluloisc/PD/Ag fabrics arising from the chemical bonds between Ag NPs and polydopamine ${ }^{26}$. Messersmith et al. reported the formation of multifunctional polymer coatings through dip-coating of some materials in the solution of dopamine. They used dopamine self-polymerization to form thin films onto inorganic and organic materials. Secondary coatings can be added to create a variety of ad-layers ${ }^{29}$. The formation of $\mathrm{Cu}$ NPs in the presence of a catalyst $\mathrm{Ag}$ NPs onto PD surface is realized and Ag NPs prevent the formation of CuO NPs. It was found that the rate of $\mathrm{Cu}$ NPs drastically increases in the presence of Ag NPs, while without the Ag NPs only a coating of the reaction vessel surface is observed ${ }^{30}$.

The new peak at $1477 \mathrm{~cm}^{-1}$ related to $\mathrm{C}=\mathrm{O}$ groups is assured to the formation of $\mathrm{Cu}$ NPs on cellulosic fabric filter paper and is due to cellulose oxidation during the reduction of $\mathrm{Cu}$ ions. Also, the peak detected at $1614 \mathrm{~cm}^{-1}$ is indicated the stretching vibration of $\mathrm{C}=\mathrm{O}$ bond in the cellulosic chain. The broad peak at $3200-3600 \mathrm{~cm}^{-1}$ relates to $\mathrm{OH}$ bond stretching vibrations. This confirms that the absorption of $\mathrm{Cu}$ NPs onto the cellulosic fabric has occurred through the physical attachment ${ }^{31}$. The final layer of $\mathrm{Cu}(\mathrm{OH})_{2}$ functionalized cellulosic filter paper produced from the oxidation of $\mathrm{Cu}$ NPs by $\mathrm{NaOH}$ and ammonium persulfate is emphasized by the IR vibrations spectrum shown in Fig. 2. C-O stretching vibrations at $938 \mathrm{~cm}^{-1}$ can be assigned to the corresponding metal cation $\left(\mathrm{Cu}^{2+}\right)$ in $\mathrm{Cu}(\mathrm{OH})_{2}$ which appears at $933 \mathrm{~cm}^{-1}$ for pure $\mathrm{Cu}(\mathrm{OH})_{2}$. The band at $750 \mathrm{~cm}^{-1}$ is attributed to the bending vibrations of $\mathrm{H}$ bonded $\mathrm{OH}$ groups. In addition, $\mathrm{Cu}-\mathrm{O}$ stretching and $\mathrm{Cu}-\mathrm{O}-\mathrm{H}$ bending vibrations have appeared at low frequencies at $610 \mathrm{~cm}^{-132-34}$.

The prepared copper hydroxide samples were also characterized by Raman spectroscopy. Raman spectroscopy is a successful apparatus for looking at the surface structure of cellulosic filter paper, so it is reasonable for investigating the surface layer of copper hydroxide NPs. Figure $1 \mathrm{~b}$ shows the Raman spectra of $\mathrm{Cu}(\mathrm{OH})_{2}$ on the cellulosic filter paper. The peak at $453 \mathrm{~cm}^{-1}$ was assigned to a Cu-O vibrational mode, while the peak at $865 \mathrm{~cm}^{-1}$ was assigned to $\mathrm{Cu}-\mathrm{O}-\mathrm{H}$ vibrations a strong and sharp band at $293 \mathrm{~cm}^{-135,36}$.

The absorption peak of the formation of Cu NPs in presence of PD appears after a long time with low intensity as shown in Fig. 1c. On the other hand, a high absorption spectrum at $324 \mathrm{~nm}$ of Cu NPs appears due to the catalytic effect of $\mathrm{Ag} \mathrm{NPs}^{37,38}$. Ag NPs as the catalytic activation seeds can catalyze Cu NPs to deposit on the surface of fabrics. In the initial stage of the reaction, $\mathrm{Cu}$ ions in the copper sulfate solution are preferentially reacted with Ag NPs due to the strong catalytic activity of $\mathrm{Ag} \mathrm{NPs}^{37,39}$. As a result, $\mathrm{Cu}$ NPs are dispersed uniformly to cover the surface of cellulosic fibers.

Figure 2 depicts XRD patterns of the pristine and modified cellulosic filter paper surfaces to determine their crystal type and crystallinity. The pure surface of cellulosic filter paper appears characteristic cellulose patterns at $14.7^{\circ}, 16.7^{\circ}, 22.7^{\circ}$, and $34.5^{\circ}$, respectively qualified to the diffraction planes of $(1-10),(110),(200)$, and (004). It is observed that the layer of PD has no effect on the crystal structure of cellulosic filter paper owing to the construction of a thin and amorphous layer of PD. The existence of Ag NPs layer is proved onto modified cellulosic filter paper/PD by the formed of the cube phase for Ag NPs at $37.7^{\circ}, 38.1^{\circ}, 44.1^{\circ}, 64.6^{\circ}$, and $77.5^{\circ}$ which are assigned to (111), (111), (200), (220), and (311) planes, respectively ${ }^{27,40}$. No diffraction peaks for Ag oxide or halides were observed, indicating that elemental Ag was present on the surface of polydopamine-coated cellulosic filter paper.

The sharp diffraction peaks at $43.7^{\circ}, 44.5^{\circ}, 50.7^{\circ}$, and $74.3^{\circ}$ concern Cu NPs and they are corresponded to (111), (111), (200), and (220) planes of face centered-cube structure, respectively. The crystalline phase of the $\mathrm{Cu}(\mathrm{OH})_{2}$ nanostructures is shown in Fig. 2. The diffraction peaks noted at $16.6^{\circ}, 23.8^{\circ}, 34.1^{\circ}, 39.7^{\circ}$, and $53.5^{\circ}$ are assigned to the (020), (021), (002), (130) and (150) planes, respectively, of orthorhombic structure and this confirms of formation high crystalline $\mathrm{Cu}(\mathrm{OH})_{2}{ }^{41}$.

Morphological properties. The SEM images and EDX of each layer synthesized on the cellulosic filter paper are illustrated in Fig. 3. The surface of cellulosic fiber has a fibers structure in the microscale range with inhomogeneous diameters from 1 to $30 \mu \mathrm{m}$ to form a network structure that seem too tight as displayed in Fig. 3a. According to the SEM image of the PD layer shown in Fig. 3b, the PD layer is transparent, uniform, and bright coated onto the cellulosic filter paper substrate. In addition, the morphology of the modified surface of cellulosic filter paper with PD/Ag depicted in Fig. 3C has been precipitated with irregular fine shapes. These particles are continuous and connected with each other. Ag NPs with about $100 \mathrm{~nm}$ are observed on a rough PD coating. The existence of the Ag NPs layer on the surface of modified FP is emphasized by the green lines presented in the EDX spectrum of Fig. 3d. 
(a)

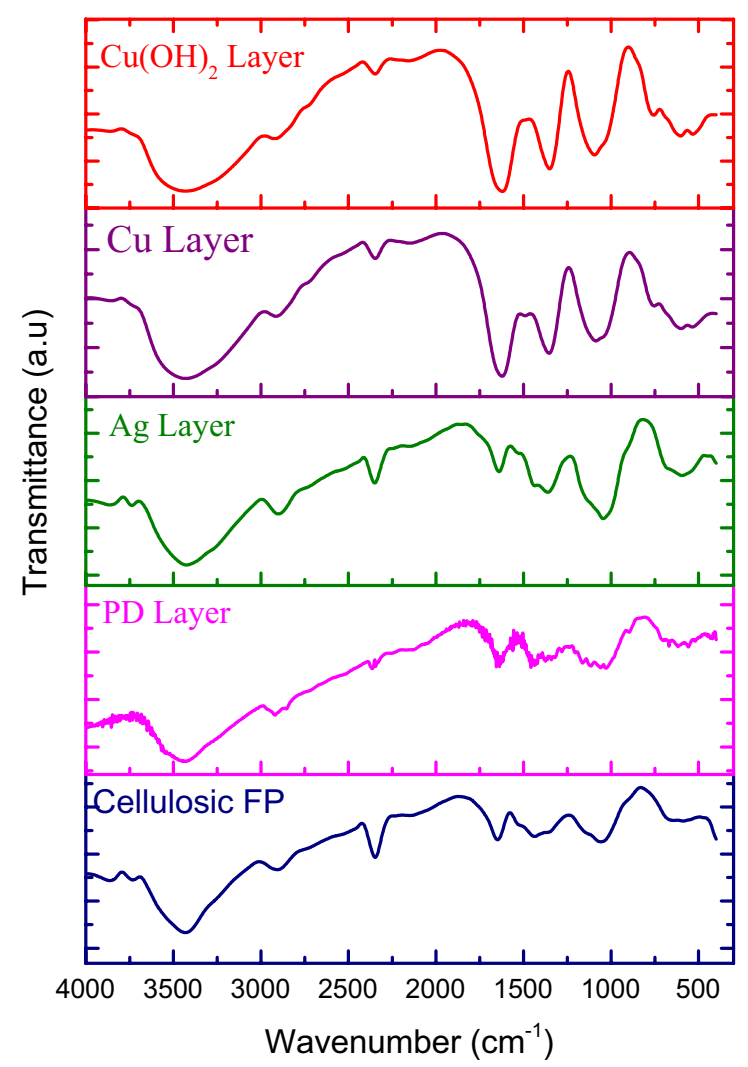

(b)

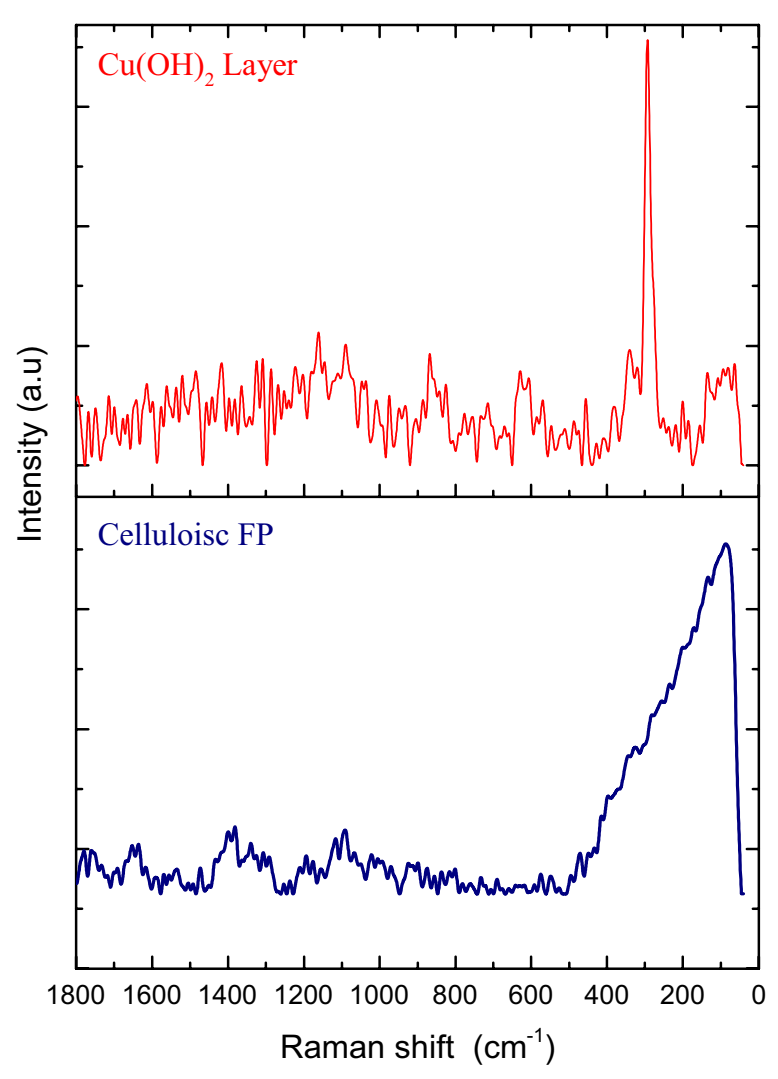

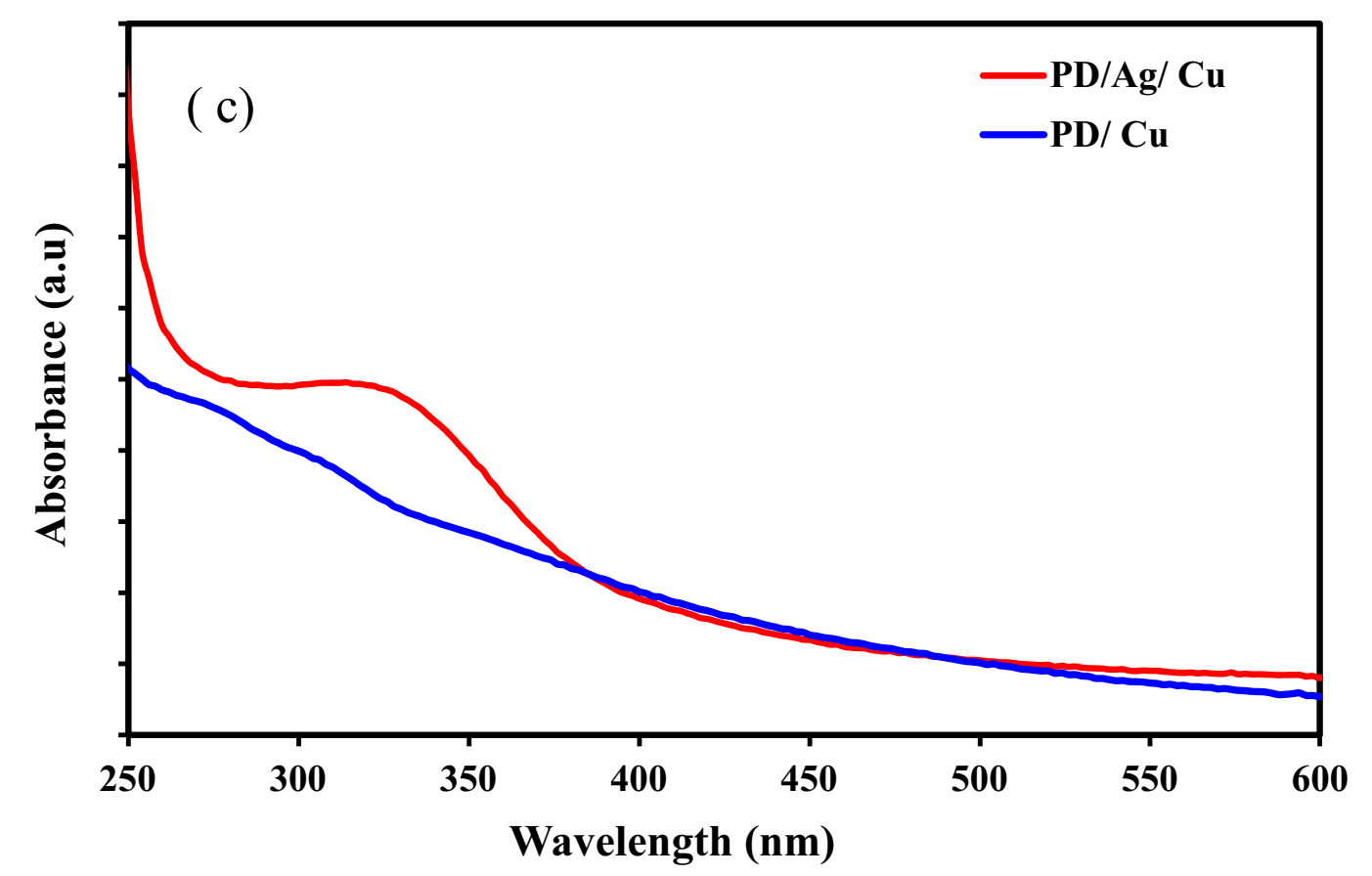

Figure 1. FTIR (a) Raman (b) spectra of cellulosic filter paper modified with different layers, (c) UV-Vis of $\mathrm{PD} / \mathrm{Ag} / \mathrm{Cu}$ and $\mathrm{PD} / \mathrm{Cu}$.

$\mathrm{Cu}$ NPs coating layer formed and shown in Fig. 3e onto the modified cellulosic filter paper with PD/Ag NPs substrate has a rough structure. It is observed that the well-covered and large $\mathrm{Cu}$ particles formed in the range of $0.5 \mu \mathrm{m}$ on the surface of cellulosic filter paper. Figure $3 \mathrm{f}$ displays the existence of $\mathrm{Cu}$ particles in the EDX 


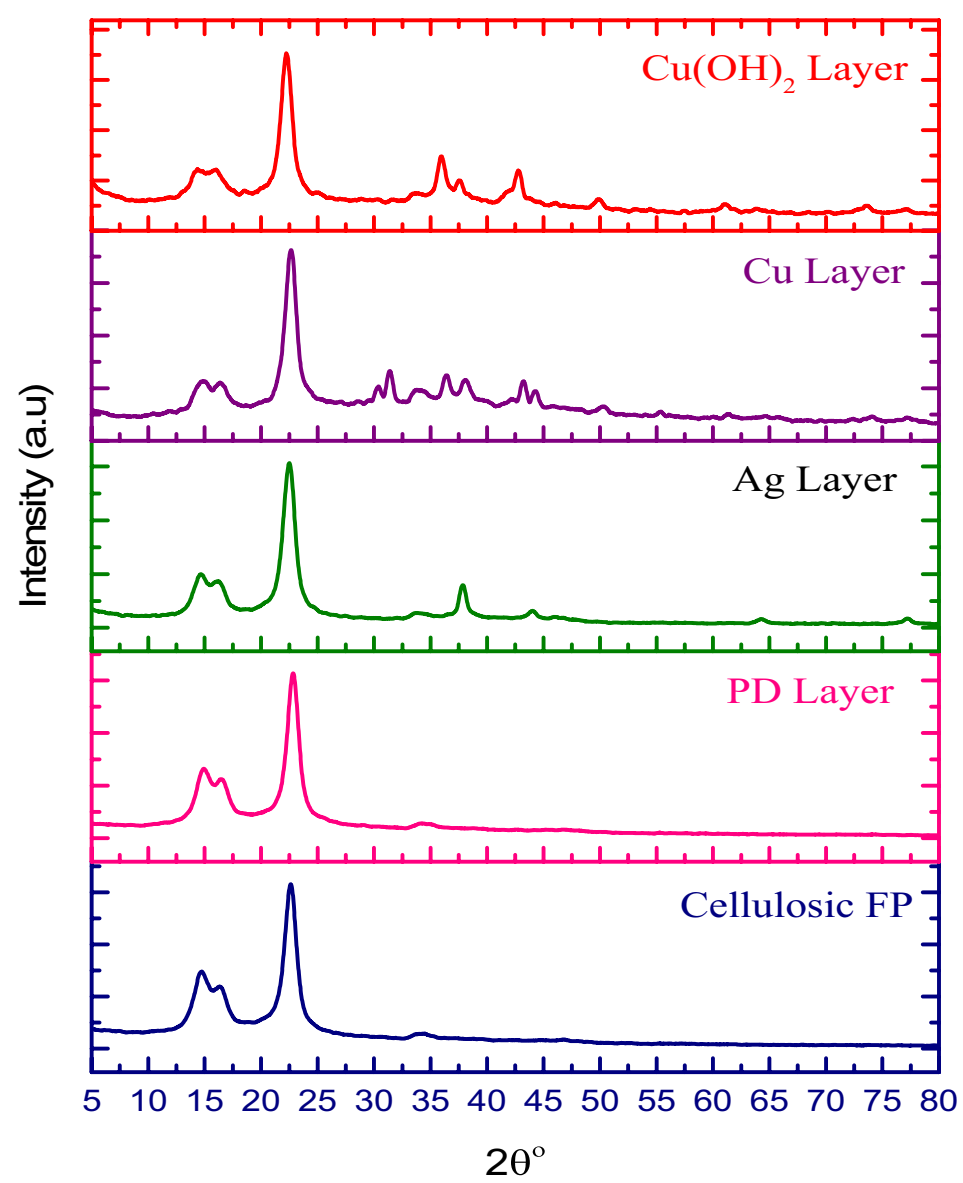

Figure 2. XRD patterns of cellulosic filter paper modified with different layers.

spectrum. Through a typical oxidizing reaction to form copper hydroxide, the $\mathrm{Cu}$ particles become and transformed into nanorods as shown in Fig. $3 \mathrm{~g}$. The presence of the copper and oxygen \% is 32.5213 to $67.4787 \%$, in the modified cellulosic filter paper as depicted in Fig. $3 \mathrm{~h}$ and it is noticed that the amount of copper is half of the amount of oxygen that proves the formation of copper hydroxide.

As shown in the HRTEM images in Fig. 4, the prepared copper hydroxide is a uniform hexagonal with mean diameters of approximately $9-39 \mathrm{~nm}$. At high magnification, the lattice space is observed to be $2 \AA$ Á. SAED pattern can be used to identify the crystal structures. There are clear diffraction rings with white spots indicating polycrystalline nanoparticles with a $5.6 \AA ̊$ lattice distance.

Hydrophobicity of the modified surface of cellulosic filter paper. Generally, the cellulosic filter paper surface is superhydrophilic due to its structure ${ }^{42}$. The $\mathrm{Cu}(\mathrm{OH})_{2}$ nanorods grown on the surfaces of the cellulosic filter paper enhance surface roughness and the n-dodecanthiol coating reduces the surface energy to increase the superhydrophobic property. The modified cellulosic filter paper is designed for oils (organic solvents) /water separation. This material will be directly in contact with mixture oil (organic solvents) and water during the service.

The three parameters that control the performance of the application of the superhydrophobic modified cellulosic filter papers in oil and water separation are the absorption capacity of the oil, separation efficiency, and water flux. The fabricated superhydrophobic functionalized cellulosic filter paper significantly absorbs the different organic solvents and some oils. Figure 5. illustrates the different range of oils and organic solvents absorption for the capacities of the modified $\mathrm{Cu}(\mathrm{OH})_{2}$ nanorods grown on the surfaces of the cellulosic filter paper. These modified filter papers have high oil and organic solvent absorption capacities. This can be elucidated based on the $\mathrm{Cu}(\mathrm{OH})_{2}$ nanorods grown onto the cellulosic filter paper is highly lipophilic and is easily wetted with the viscous oils. The oil absorption capacities values are 3.4, 4.4, 4.5, 4.7, 5.2, 6.1, 6.4, 7.2, and $8.2(\mathrm{~g} / \mathrm{g})$ for $\mathrm{n}$-hexane, dichloromethane, chloroform, toluene, diesel, sunflower oil, olive oil, waste engine oil, and diesel oil, respectively.

The separation efficiency and the oil flux of oil or organic solvents are measured and calculated and the separation efficiency using the modified cellulosic filter paper is higher than $99 \%$ for the oil/water mixtures. From Fig. 6, it is observed that the separation efficiency of the modified filter paper is higher than $99 \%$ and maintains this value after 100 cycles. The values of these efficiencies are found to be $99.68 \%, 99.74 \%, 99.84 \%$, 99.9\%, 99.57\%, 99.5\%, 99.36\%,99.1\%, and 99.2\% for chloroform, dichloromethane, toluene, n-hexane, diesel, sunflower oil, olive oil, waste engine oil and diesel oil, respectively. In addition, Fig. 7a. presents high values of 

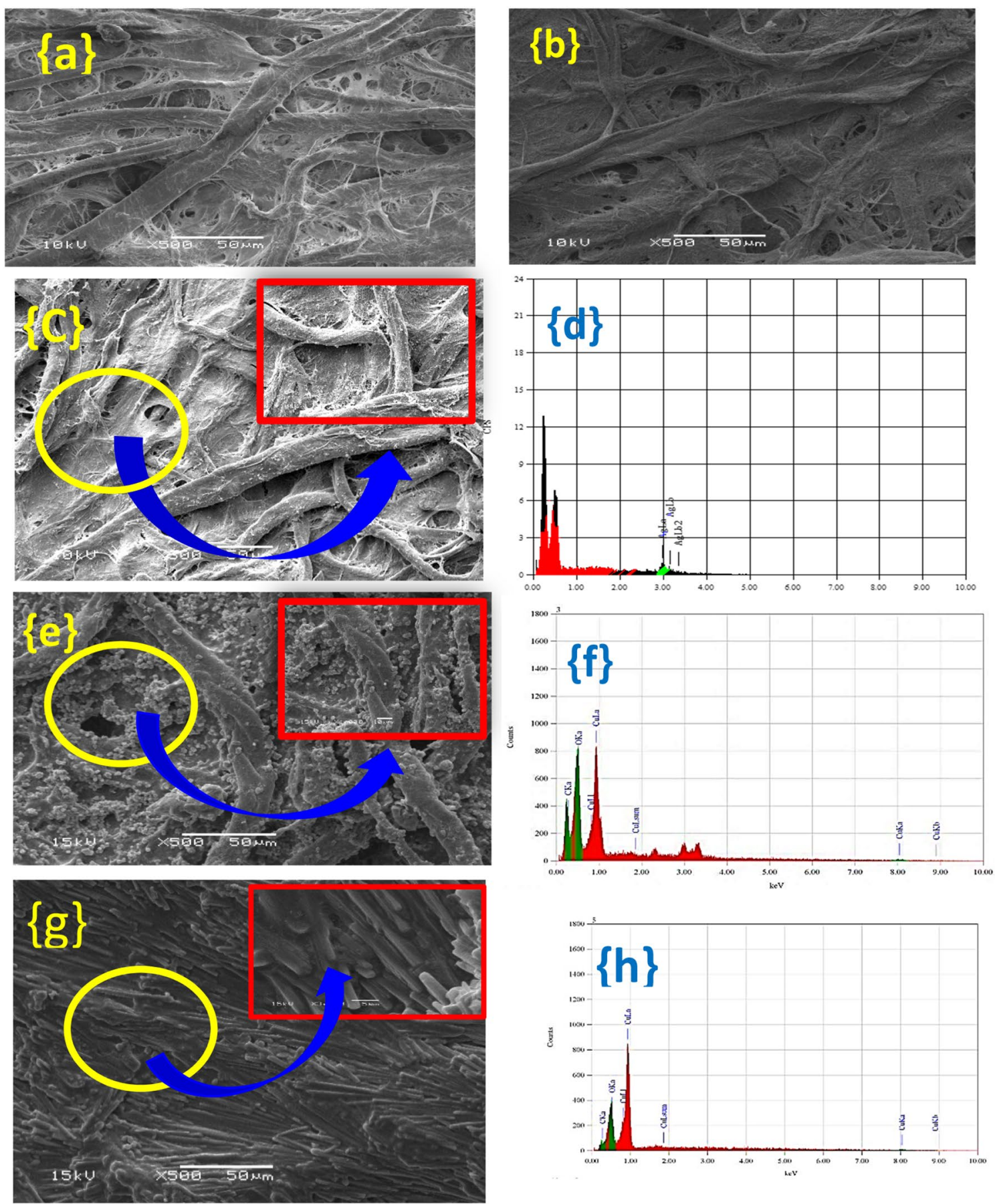

Figure 3. SEM images of modified cellulosic FP surfaces with different layers (a) Pure cellulosic FP, (b) PD layer, (c) Ag NPs layer, (e) Cu NPs layer, and $(\mathbf{g}) \mathrm{Cu}(\mathrm{OH})_{2}$ nanorods layer. EDX analysis of $(\mathbf{d}) \mathrm{Ag}$ layer, $(\mathbf{f}) \mathrm{Cu}$ layer and $(\mathbf{h}) \mathrm{Cu}(\mathrm{OH})_{2}$ layer.

the permeate flux values for the different oils and organic solvents through the $\mathrm{Cu}(\mathrm{OH})_{2}$ nanorods modified filter paper. This is cleared for the low viscous solvents and the permeate flux values are5876, 8105.2, 8375.7, and 9663.5 $\mathrm{Lm}^{-2} \mathrm{~h}^{-1}$ for chloroform, dichloromethane, toluene, and $\mathrm{n}$-hexane, respectively. In addition, the oil flux values for the viscous oils such as diesel, sunflower, olive, waste engine, and diesel oils are 1452.7, 284.7, 236.8, 166.3 , and $150.8 \mathrm{Lm}^{-2} \mathrm{~h}^{-1}$ respectively as observed in Fig. $7 \mathrm{~b}$. The value of flux mainly depends on the viscosity of different oils. To measure the water content in the oil, we used Karl Fisher coulometric titration. Karl Fischer analysis for water content can yield highly accurate results. It was detected the water content in the oil before and after the oil-water separation process for $\mathrm{n}$-hexane as low viscous oil and diesel as high viscous oil. We notice that the water contents for $n$-hexane and diesel are $0.01 \%$ and $0.02 \%$, respectively. After the separation process, the water contents are to be $0.02 \%$ and $0.03 \%$ for $n$-hexane and diesel, respectively. 

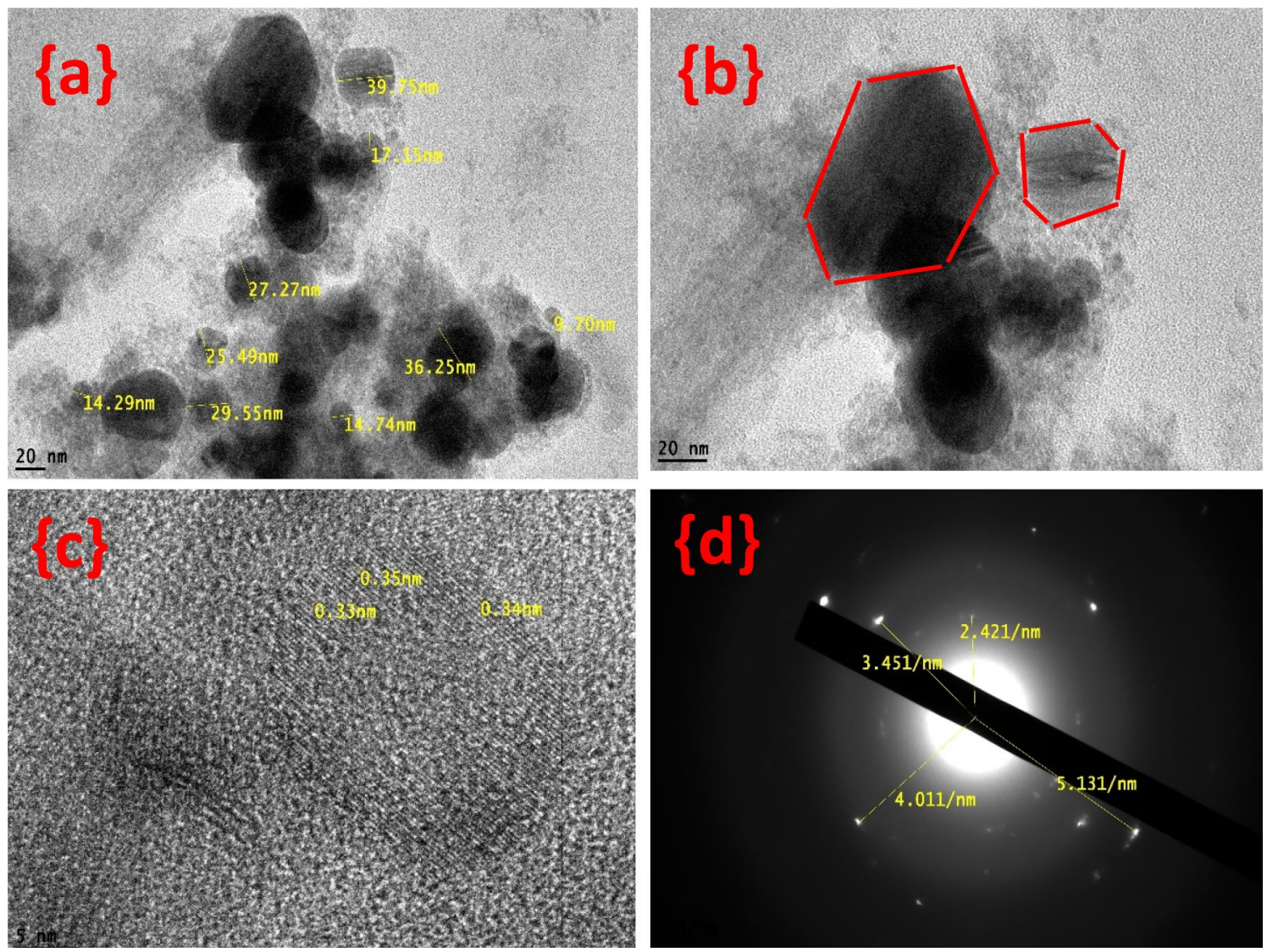

Figure 4. HRTEM images of copper hydroxide at different magnifications (a, b) and the SAED (c, d).

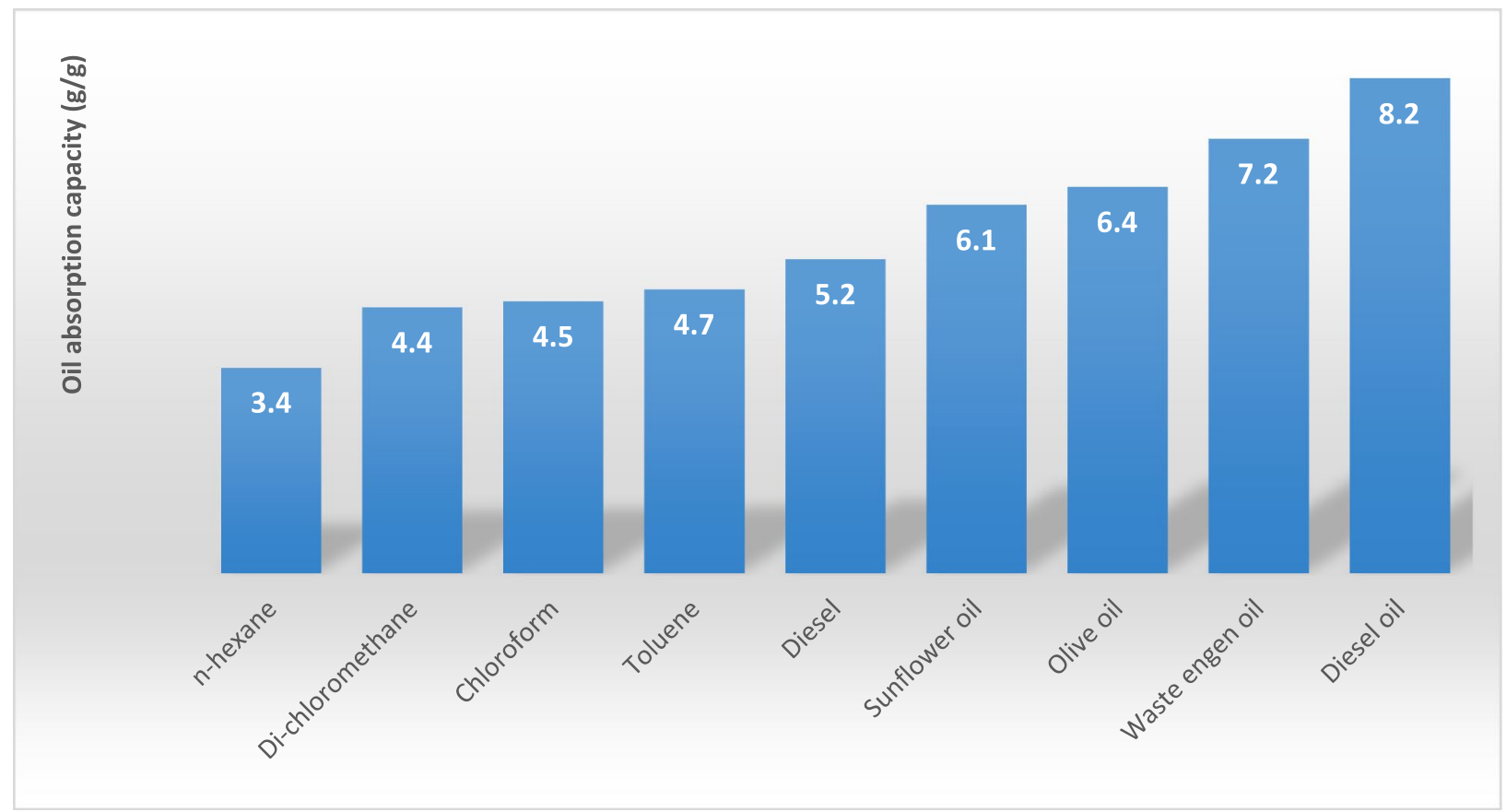

Figure 5. Absorption capacities of the modified surface of cellulosic filter papers in different oils and organic solvents. 


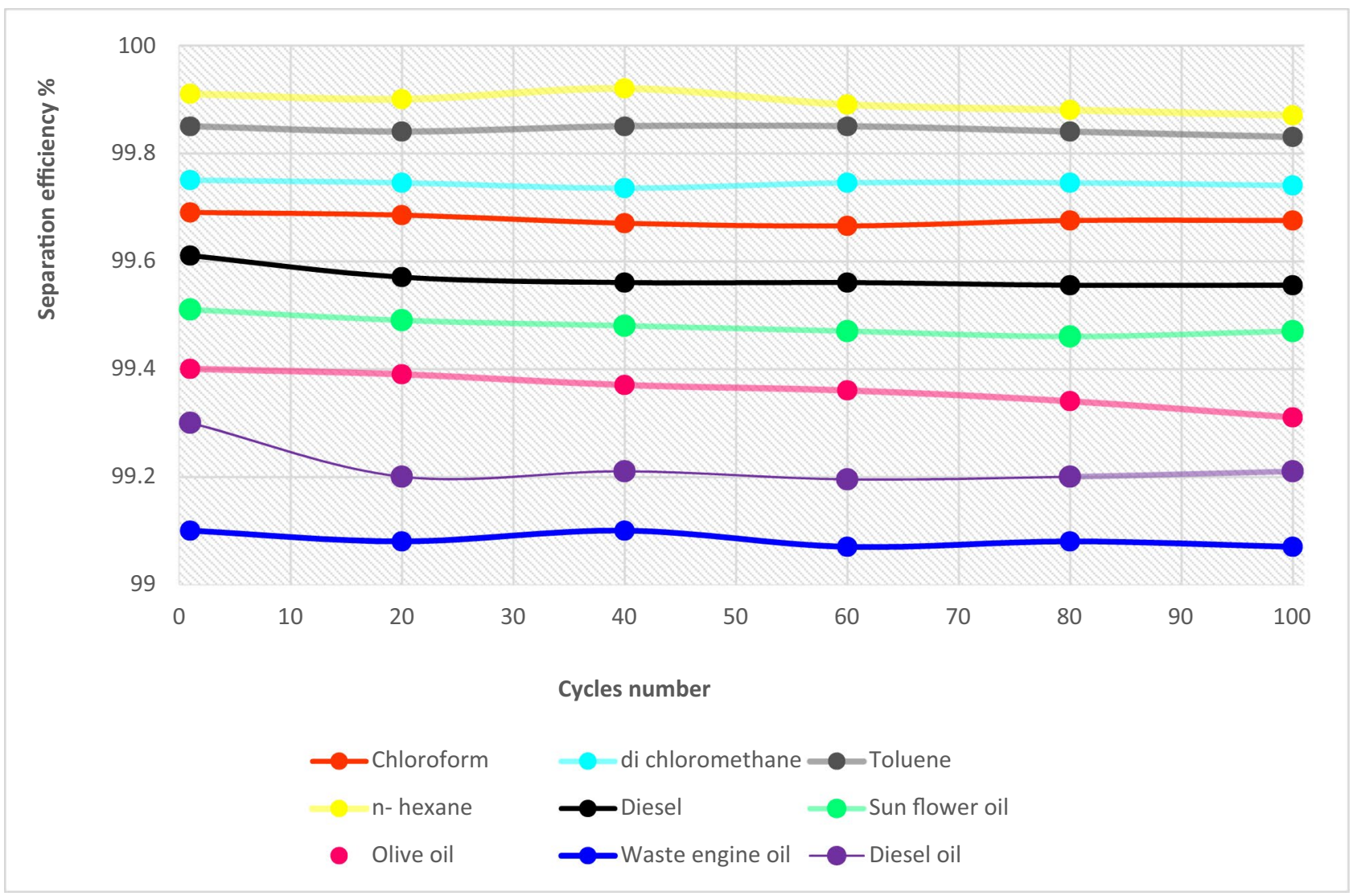

Figure 6. Separation efficiency of modified cellulosic FP membranes for different oils and solvents.

\section{Conclusions}

Converting the surface of the cellulosic paper from superhydrophilic to superhydrophobic using immersion technique was investigated by deposition of $\mathrm{Cu}(\mathrm{OH})_{2}$ nanorods then coating this rough surface with $n$-dodecanthiol. The water contact angle of the modified surface of the cellulosic paper is $169.7^{\circ}$. The superphydrophobic modified cellulosic papers possess good stability in both water and oil, which indicates that they can be used as a stable oil/water separation material. The modified surfaces of cellulosic papers had a high absorption capacity for diesel oil is $8.2(\mathrm{~g} / \mathrm{g})$. The separation efficiency for different oil/water mixtures was higher than $99 \%$ and the flux of this modified cellulosic paper for low viscous n-hexane and high viscous diesel oil were 9663.5 and $150.8 \mathrm{~L} \mathrm{~m}^{-2} \mathrm{~h}^{-1}$, respectively. It was detected that these modified surfaces of cellulosic paper were stable for 100 cycles and one month. 

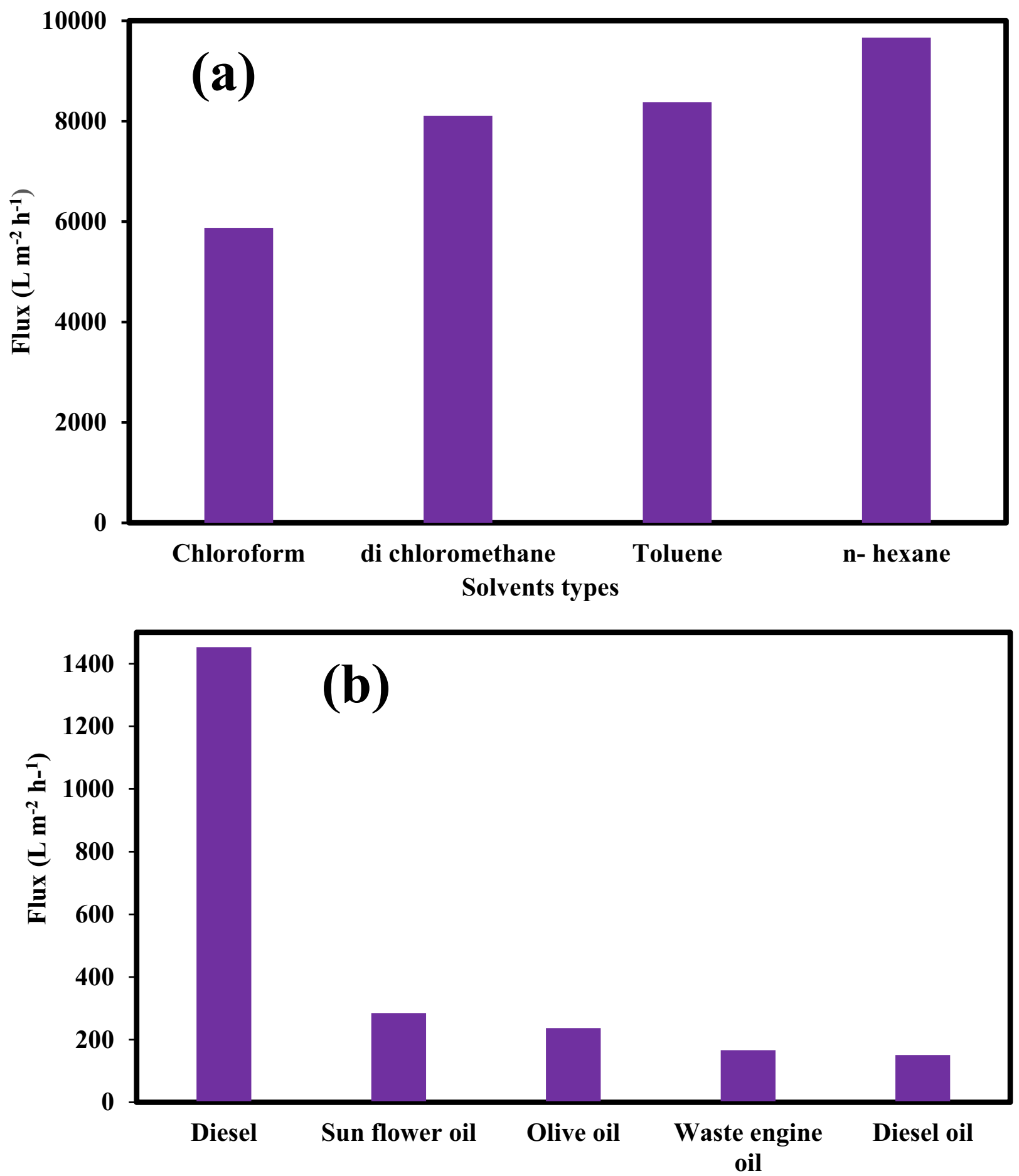

Oils types

Figure 7. Flux of the modified surface cellulosic filter papers (a) low viscous and (b) high viscous organic solvents.

Received: 17 May 2021; Accepted: 29 July 2021

Published online: 10 August 2021

\section{References}

1. Corneliu, N. (Google Patents, 1968).

2. He, H. et al. Hierarchical WO3@ Cu (OH) 2 nanorod arrays grown on copper mesh with superwetting and self-cleaning properties for high-performance oil/water separation. J. Alloy. Compd. 855, 157421. https://doi.org/10.1016/j.jallcom.2020.157421 (2021).

3. Giang, H. N. et al. Fabrication of superhydrophobic surface using one-step chemical treatment. Surfaces Interfaces 21, 100673. https://doi.org/10.1016/j.surfin.2020.100673 (2020). 
4. Gupta, R. K., Dunderdale, G. J., England, M. W. \& Hozumi, A. J. J. O. M. C. A. Oil/water separation techniques: A review of recent progresses and future directions. Journal of Materials Chemistry A 5, 16025-16058 (2017).

5. Luo, J. et al. Recent advances in separation applications of polymerized high internal phase emulsions. J. Sep. Sci. 44, 169-187. https://doi.org/10.1002/jssc.202000612 (2021).

6. Lv, P. et al. Stretchable Textiles with Superwettabilities for Tunable Oil-Water Separation. ChemNanoMat 6, 1111-1118. https:// doi.org/10.1002/cnma.202000178 (2020).

7. Ruan, X., Xu, T., Chen, D., Ruan, Z. \& Hu, H. Superhydrophobic paper with mussel-inspired polydimethylsiloxane-silica nanoparticle coatings for effective oil/water separation. RSC Adv. 10, 8008-8015. https://doi.org/10.1039/C9RA08018J (2020).

8. Ebrahim, S., Shokry, A., Khalil, M., Ibrahim, H. \& Soliman, M. J. S. R. Polyaniline/Ag nanoparticles/graphene oxide nanocomposite fluorescent sensor for recognition of chromium (VI) ions. Sci. Rep. 10, 1-11. https://doi.org/10.1038/s41598-020-70678-8 (2020).

9. Cheng, Z. et al. $\mathrm{pH}$-controllable on-demand oil/water separation on the switchable superhydrophobic/superhydrophilic and underwater low-adhesive superoleophobic copper mesh film. Langmuir 31, 1393-1399. https://doi.org/10.1021/la503676a (2015).

10. Ke, Q., Jin, Y., Jiang, P. \& Yu, J. J. L. Oil/water separation performances of superhydrophobic and superoleophilic sponges. Langmuir 30, 13137-13142. https://doi.org/10.1021/la502521c (2014).

11. Jiang, F. \& Hsieh, Y.-L. Amphiphilic superabsorbent cellulose nanofibril aerogels. J. Mater. Chem. A 2, 6337-6342. https://doi.org/ 10.1039/C4TA00743C (2014).

12. Ceylan, D. et al. Evaluation of butyl rubber as sorbent material for the removal of oil and polycyclic aromatic hydrocarbons from seawater. Environ. Sci. Technol. 43, 3846-3852. https://doi.org/10.1021/es900166v (2009).

13. Xu, X., Li, M., Li, X., Zhang, L. J. J. O. I. \& Chemistry, E. Fabricated smart sponge with switchable wettability and photocatalytic response for controllable oil-water separation and pollutants removal. J. Ind. Eng. Chem. 92, 278-286. https://doi.org/10.1016/j. jiec.2020.09.017 (2020).

14. Sidik, S. et al. Modified oil palm leaves adsorbent with enhanced hydrophobicity for crude oil removal. Chem. Eng. J. 203, 9-18. https://doi.org/10.1016/j.cej.2012.06.132 (2012).

15. Karatum, O., Steiner, S. A. III., Griffin, J. S., Shi, W. \& Plata, D. Flexible, mechanically durable aerogel composites for oil capture and recovery. ACS Appl. Mater. Interfaces. 8, 215-224. https://doi.org/10.1021/acsami.5b08439 (2016).

16. Yang, Y., Liu, Z., Huang, J. \& Wang, C. Multifunctional, robust sponges by a simple adsorption-combustion method. J. Mater. Chem. A 3, 5875-5881. https://doi.org/10.1039/C5TA00454C (2015).

17. Zhang, W. et al. A solvothermal route decorated on different substrates: Controllable separation of an oil/water mixture to a stabilized nanoscale emulsion. Adv. Mater. 27, 7349-7355. https://doi.org/10.1002/adma.201502695 (2015).

18. Belal, A. S., Khalil, M., Soliman, M. \& Ebrahim, S. Novel superhydrophobic surface of cotton fabrics for removing oil or organic solvents from contaminated water. Cellulose 27, 7703-7719. https://doi.org/10.1007/s10570-020-03316-1 (2020).

19. Shokry, A., Khalil, M., Ibrahim, H., Soliman, M. \& Ebrahim, S. J. S. R. Acute toxicity assessment of polyaniline/Ag nanoparticles/ graphene oxide quantum dots on Cypridopsis vidua and Artemia salina. Sci. Rep. 11, 1-9. https://doi.org/10.1038/s41598-02184903-5 (2021).

20. Fan, T., Qian, Q., Hou, Z., Liu, Y. \& Lu, M. Preparation of smart and reversible wettability cellulose fabrics for oil/water separation using a facile and economical method. Carbohydr. Polym. 200, 63-71. https://doi.org/10.1016/j.carbpol.2018.07.040 (2018).

21. Chien, H.-W., Tsai, M.-Y., Kuo, C.-J. \& Lin, C.-L. Well-dispersed silver nanoparticles on cellulose filter paper for bacterial removal. Nanomaterials 11, 595. https://doi.org/10.3390/nano11030595 (2021)

22. Rosa, M. et al. Cellulose nanowhiskers from coconut husk fibers: effect of preparation conditions on their thermal and morphological behavior. Carbohyd. Polym. 81, 83-92. https://doi.org/10.1016/j.carbpol.2010.01.059 (2010).

23. Poletto, M., Pistor, V., Zeni, M. \& Zattera, A. Crystalline properties and decomposition kinetics of cellulose fibers in wood pulp obtained by two pulping processes. Polym. Degrad. Stab. 96, 679-685. https://doi.org/10.1016/j.polymdegradstab.2010.12.007 (2011).

24. Hospodarova, V., Singovszka, E. \& Stevulova, N. Characterization of cellulosic fibers by FTIR spectroscopy for their further implementation to building materials. Am. J. Anal. Chem. 9, 303-310. https://doi.org/10.4236/ajac.2018.96023 (2018).

25. Xu, Z., Miyazaki, K. \& Hori, T. Fabrication of polydopamine-coated superhydrophobic fabrics for oil/water separation and selfcleaning. Appl. Surf. Sci. 370, 243-251. https://doi.org/10.1016/j.apsusc.2016.02.135 (2016).

26. Kim, H. W. et al. Oxygen concentration control of dopamine-induced high uniformity surface coating chemistry. ACS Appl. Mater. Interfaces 5, 233-238. https://doi.org/10.1021/am302439g (2013).

27. Niyonshuti, I. I. et al. Polydopamine surface coating synergizes the antimicrobial activity of silver nanoparticles. ACS Appl. Mater. Interfaces 12, 40067-40077. https://doi.org/10.1021/acsami.0c10517 (2020).

28. Thota, R. \& Ganesh, V. Simple and facile preparation of silver-polydopamine (Ag-PDA) core-shell nanoparticles for selective electrochemical detection of cysteine. RSC Adv. 6, 49578-49587. https://doi.org/10.1039/C6RA06994K (2016).

29. Lee, H., Dellatore, S. M., Miller, W. M. \& Messersmith, P. B. J. S. Mussel-inspired surface chemistry for multifunctional coatings. 318, 426-430. https://doi.org/10.1126/science.1147241 (2007).

30. Grouchko, M., Kamyshny, A., Ben-Ami, K. \& Magdassi, S. J. J. O. N. R. Synthesis of copper nanoparticles catalyzed by pre-formed silver nanoparticles. 11, 713-716. https://doi.org/10.1007/s11051-007-9324-5 (2009).

31. Sedighi, A., Montazer, M. \& Hemmatinejad, N. Copper nanoparticles on bleached cotton fabric: in situ synthesis and characterization. Cellulose 21, 2119-2132. https://doi.org/10.1007/s10570-014-0215-5 (2014).

32. Zhang, Y. X., Huang, M., Li, F. \& Wen, Z. Q. Controlled synthesis of hierarchical CuO nanostructures for electrochemical capacitor electrodes. Int. J. Electrochem. Sci 8, 8645-8661 (2013).

33. Gregorio-Jauregui, K. M. et al. One-step method for preparation of magnetic nanoparticles coated with chitosan. J. Nanomater. 2012. https://doi.org/10.1155/2012/813958 (2012).

34. Gao, D., Wang, L., Wang, C. \& Chen, T. Photocatalytic self-cleaning cotton fabrics coated by $\mathrm{Cu}_{2}(\mathrm{OH}) \mathrm{PO}_{4}$ under Vis/NIR irradiation. Materials 12, 238. https://doi.org/10.3390/ma12020238 (2019).

35. Aguirre, J. M., Gutiérrez, A. \& Giraldo, O. Simple route for the synthesis of copper hydroxy salts. J. Braz. Chem. Soc. 22, 546-551. https://doi.org/10.1590/S0103-50532011000300019 (2011).

36. Bulakhe, R. N. et al. Chemically grown 3D copper hydroxide electrodes with different morphologies for high-performance asymmetric supercapacitors. J. Ind. Eng. Chem. 66, 288-297. https://doi.org/10.1016/j.jiec.2018.05.043 (2018).

37. Díaz-Visurraga, J. et al. Study on antibacterial alginate-stabilized copper nanoparticles by FT-IR and 2D-IR correlation spectroscopy. 7, 3597. https://doi.org/10.2147/IJN.S32648 (2012).

38. Foresti, E. et al. New thiophene monolayer-protected copper nanoparticles: Synthesis and chemical-physical characterization. 2008. https://doi.org/10.1155/2008/649130 (2008).

39. Wang, K., Ma, Q., Zhang, Y., Wang, S. \& Han, G. J. P. Ag NPs-assisted synthesis of stable Cu NPs on PET fabrics for antibacterial and electromagnetic shielding performance. 12, 783. https://doi.org/10.3390/polym12040783 (2020).

40. Shokry, A., Khalil, M., Ibrahim, H., Soliman, M. \& Ebrahim, S. J. S. R. Highly luminescent ternary nanocomposite of polyaniline, silver nanoparticles and graphene oxide quantum dots. Sci. Rep. 9, 1-12. https://doi.org/10.1038/s41598-019-53584-6 (2019).

41. He, D. et al. Facile route to achieve mesoporous $\mathrm{Cu}(\mathrm{OH})_{2}$ nanorods on copper foam for high-performance supercapacitor electrode. J. Alloys Compd. 699, 706-712. https://doi.org/10.1016/j.jallcom.2016.12.398 (2017).

42. Teisala, H., Tuominen, M. \& Kuusipalo, J. J. A. M. I. Superhydrophobic coatings on cellulose-based materials: Fabrication, properties, and applications. Adv. Mater. Interfaces 1, 1300026. https://doi.org/10.1002/admi.201300026 (2014). 


\section{Acknowledgements}

The authors thank the Nanotechnology and Composite Materials Department, Institute of New Materials and Advanced Technology, City of Scientific Research and Technological Applications, for help.

\section{Author contributions}

A.S.B. and M.K. contributed to ideas, experiment execution, and interpretation of the data and writing of the manuscript. J.E.N. and A.S. share in the characterization and explaining the results of characterization. S.E. and M.S. supervised the experiments and wrote and edited the manuscript.

\section{Competing interests}

The authors declare no competing interests.

\section{Additional information}

Correspondence and requests for materials should be addressed to M.K.

Reprints and permissions information is available at www.nature.com/reprints.

Publisher's note Springer Nature remains neutral with regard to jurisdictional claims in published maps and institutional affiliations.

(c) (i) Open Access This article is licensed under a Creative Commons Attribution 4.0 International License, which permits use, sharing, adaptation, distribution and reproduction in any medium or format, as long as you give appropriate credit to the original author(s) and the source, provide a link to the Creative Commons licence, and indicate if changes were made. The images or other third party material in this article are included in the article's Creative Commons licence, unless indicated otherwise in a credit line to the material. If material is not included in the article's Creative Commons licence and your intended use is not permitted by statutory regulation or exceeds the permitted use, you will need to obtain permission directly from the copyright holder. To view a copy of this licence, visit http://creativecommons.org/licenses/by/4.0/.

(C) The Author(s) 2021 